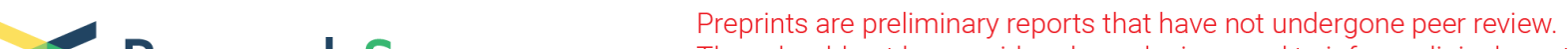 (2) \\ YYFZBJS Inhibits Colorectal Tumorigenesis by Remodeling Enterotoxigenic Bacteroides Fragilis Mediated M2 Macrophage Polarization in Vivo and in Vitro
}

\section{Ni Chai}

Shanghai University of Traditional Chinese Medicine Yueyang Hospital of Integrated Traditional Chinese Medicine and Western Medicine

\section{Yuelei Cheng}

Shuguang hospital

\section{Yibai Xiong}

China Academy of Chinese Medical Sciences

\section{Wenfei Shi}

Shanghai Municipal Institute Hospital of Traditional Chinese Medicine

\section{Yiqing Yao}

Shanghai University of Traditional Chinese Medicine Yueyang Hospital of Integrated Traditional Chinese Medicine and Western Medicine

\section{Limei Yang}

Shuguang Hospital

Hua Sui ( $\square$ huasui0808@shutcm.edu.cn )

Shuguang Hospital

\section{Huirong Zhu}

Shanghai University of Traditional Chinese Medicine

\section{Research}

Keywords: colorectal cancer, ETBF, AOM/DSS-induced colitis-associated colorectal cancer, macrophage, signal pathway

Posted Date: December 15th, 2020

DOl: https://doi.org/10.21203/rs.3.rs-125313/v1

License: (c) (1) This work is licensed under a Creative Commons Attribution 4.0 International License. Read Full License 


\section{Abstract}

\section{Background}

The occurrence of CRC is believed to be related to a variety of factors. Accumulating evidence shows that microbiota can influence the outcome of cancer immunotherapy. Our previous studies indicated that the extract of Yi-Yi-Fu-Zi-Bai-Jiang-San (YYFZBJS), had potent anticancer activities by significantly inhibiting intestinal tumor development in $\mathrm{Ap}^{\mathrm{Min} /+}$ mice. However, knowledge regarding the mechanism and effect of YYFZBJS in the prevention of colorectal cancer is limited.

\section{Methods}

In this study, we investigated the preventive effects of oral administration of YYFZBJS in enterotoxigenic Bacteroides fragilis (ETBF)-colonized mice with azoxymethane (AOM)/dextran sulfate sodium (DSS)induced tumorigenesis. Here, the tumor load, tumor number, histology, and the severity of disease activity index (DAl) scores were reduced as expected.

\section{Results}

the intragastric administration of YYFZBJS in AOM/DSS model significantly decreased ETBF abundance, immunity and some M2 macrophage markers, including CD206, Arg-1, IL-10, and TGF- $\beta$. Additionally, reversing polarized macrophages, which has been modified by YYFZBJS, could suppressed CRC cell proliferation and infiltration, as demonstrated by decreasing some tumor proliferation-related proteins in a dose-dependent manner, including c-Met, cyclinD1 and MMPs. Importantly, ETBF dysbiosis can contribute to the development of colon tumor by stimulating p-STAT3 medicated M2 macrophages polarization to promote chronic inflammation and adenoma malignant transformation, which effectively constrained by YYFZBJS.

\section{Conclusion}

Altogether, we demonstrate that ETBF dysbiosis may contribute to M2 macrophages-promoted colon carcinogenesis and progression of CRC cells, and indicating that YYFZBJS could be employed as a promising protective agent against ETBF-mediated colorectal cancer.

\section{Background}

The recent rapid increase in colorectal cancer (CRC) incidence is a serious health problem worldwide, as it has one of the highest rates of cancer-related mortality [1]. Colorectal cancer usually develops from adenomatous polyps (adenoma) that undergo dysplastic changes to become cancerous (adenocarcinoma) [2]. However, the development of CRC remains to be completely understood owing to its complexity. Recently, accumulating evidence suggests that the gut microbiota, chronic inflammation, host genetic predisposition, and environmental factors have been linked with the progression of CRC [3, 4] We have demonstrated that enterotoxigenic Bacteroides fragilis (ETBF) strains secrete the $B$. fragilis 
toxin, severely altered Tregs mediated by YYFZBJS repressed CRC cancer cell growth, along with reduction of the phosphorylation of $\beta$-catenin [5].

Additionally, accumulating evidence have shown that ETBF is significantly higher in individuals with spontaneous CRC and in individuals from familial adenomatous polyposis families [6-8]. Moreover, a series of studies have demonstrated that the expansion of Treg in the colonic lamina propria lymphocytes (LPL) of ETBF-colonized $\mathrm{ApC}^{\mathrm{Min} / \mathrm{+}}$ mice is driven by tissue-resident macrophages $[9,10]$.

Moreover, these and other studies, including those from our laboratory, revealed an equally critical role for T-regulatory cells (Treg) in driving tumor growth in the ETBF-colonized gut $[11,12]$. Usually, $A p C^{M i n /+}$ mice are often utilized to elucidate mechanisms of intestinal tumorigenesis in vivo;another carcinogen-induced azoxymethane/dextran sulfate sodium (AOM/DSS) model of colon cancer are widely used to investigate the roles of dietary factors in tumor development $[13,14]$, and all polyps in AOM/DSS-treated mice form in the colon. In the current study, we assessed the tumorigenic potential of ETBF colonization in the germfree (GF) mediated AOM/DSS mouse model. We found that ETBF colonization in GF mice administered with AOM/DSS resulted in a rapid development of a large number of polyps predominantly in the colorectal region compared with wild-type GF mice.

In tumor microenvironment, tumor-secreted chemokines and growth factors could induce monocytes recruitment and differentiation to macrophage [15]. With the influence of those cytokines' signals, TAMs (tumor-associated macrophages) undergo polarization into M1 and M2 phenotypes which could promote tumor metastasis [16]. The main M2 markers include CD206, Arg-1, IL-10, and TGF- $\beta$. The main M1 markers include CD11b, iNOS, IL-12, and TNF-a [17]. Increased evidences have shown, the high density of M2 macrophages is related to shorter survival time and a high risk of recurrence in a clinical study [18, 19]. A series of studies above confirmed that $M 2$ macrophages play an important role in promoting $C R C$ growth and invasion [20]. Our research also confirmed the important role of M2 macrophages in promoting the occurrence of intestinal tumor in the AOM/DSS model [16].

The results of those studies suggest that the modulation of microbiota might be a good strategy to prevent or cure $\mathrm{CRC}$, and uncovering how symbionts suppress inflammatory response warrants further investigation [21]. Clearance of ETBF by cefoxitin, a semi-synthetic and broad-spectrum cepha antibiotic, inhibited ETBF-promoted tumorigenesis in Min mice [22]. However, there exist no studies investigating the suppressive effects of natural product or TCM on ETBF-mediated tumorigenesis in mice. Previous research has demonstrated health-promoting properties of various dietary natural compounds, including carotenoids, flavonoids, and polyphenols contained in vegetables and fruits [23]. However, few chemotherapeutic drugs are available, and such drugs have significant toxic side effects that can severely impair the immune system and hematopoietic system [24-26]. Our previous studies have shown that Yi-Yi-Fu-Zi-Bai-Jiang-San (YYFZBJS) volunteers altered dysregulated inflammation and oncogenic pathways and inhibited intestinal tumorigenesis [5]. 
In the present study, we investigated the effect of YYFZBJS in carcinogen-induced AOM/DSS model of colon cancer and the ETBF enrichment in gut microbiota, to evaluate the mechanism of preventing ETBFmediated tumorigenesis. Furthermore, gavaging germ-free AOM/DSS mice with ETBF strains, we demonstrated that ETBF accelerate colonic tumorigenesis development, partly by modulating the polarization of M2 macrophages. Furthermore, we examined the alteration of M2 macrophages polarization in GF/AOM/DSS mouse tumor tissue after YYFZBJS treatment and the efficacy and associated mechanisms of ETBF and YYFZBJS combined utilization on intestinal tumor development both in vitro and in vivo. It will help us to have a better understanding about the molecular biological mechanism in YYFZBJS and its anticancer effect, contributing to its further application in the prevention of CRC.

\section{Materials And Methods}

\section{Cell culture and reagents}

Mice colorectal adenocarcinoma MC-38 cell were purchased from the Shanghai Cell Collection (Shanghai, China). It was cultured in RPMI 1640 with 10\% fetal bovine serum (Gibco, NY, USA), 2 mM glutamine, 100 units/ml streptomycin and penicillin (Invitrogen, Carlsbad, CA). The cells were grown at $37^{\circ} \mathrm{C}$ in a humidified $5 \% \mathrm{CO}_{2}$ atmosphere. Bone marrow-derived macrophages (BMDMs) were prepared as previously described [27]. BMDMs were stimulated with IL-4 $(100 \mathrm{ng} / \mathrm{ml}, 48 \mathrm{~h})$ for $\mathrm{M} 2$ polarized activation. Monoclonal antibodies specific for JNK (3708), NFK-B (3036), c-Met (3127), cyclinD1 (4668s), MMP-2 (4668s), MMP-9 (4668s), STAT3 (9139), p-STAT3 (9145s) and GAPDH (5174s) were obtained from Abcam plc (Cambridge, UK) and Cell Signaling Technology Inc. (Boston, MA, USA).

\section{Induction of AOM-initiated and DSS-promoted colon carcinogenesis}

In total, 6-week-old SPF female C57BL/6J mice were obtained from Shanghai Super-B\&K Animal Laboratory Co., Ltd. (Shanghai, China) with Certification No. SCXK 2016-0016. All animals were housed under specific pathogen-free conditions in accordance with the provision and general recommendation of the Chinese Experimental Animals Administration Legislation. The procedures used for the induction of the CAC model by AOM and DSS as previously described [16]. Briefly, on day 1, the mice were injected with AOM (12.5 mg/kg, i.p.). After 1 week, 2.5\% DSS (International Lab, Chicago, IL, USA) was added to the drinking water for 7 days, followed by 14 days of tap water for recovery. This cycle was repeated twice. As shown in Fig. 1A, the intragastric administration of YYFZBJS-L/M/H were taken at the doses of $3.825 \mathrm{~g} / \mathrm{kg}, 7.65 \mathrm{~g} / \mathrm{kg}$ and $15.3 \mathrm{~g} / \mathrm{kg}$ according to HED (human equivalent dose) [5, 28]. YYFZBJS-L/M/H and Aspirin ( $30 \mathrm{mg} / \mathrm{kg}$ ) were given orally for 9 weeks during the DSS treatment separately as previously described (1). Aspirin is taken as a positive control, because it showed effectively inhibiting effect on intestinal adenomatous polyposis and colon carcinogenesis $[5,16]$. Control group was oral gavaged with the same volume of sterile isotonic saline and fed with normal drinking water. 


\section{Mouse strains and breeding}

As shown in Fig. 3A, mice were treated for 4 weeks with an antibiotic solution (Abx) containing Ampicillin $(1 \mathrm{mg} / \mathrm{ml})$, Neomycin $(1 \mathrm{mg} / \mathrm{mL})$, Metronidazole $(1 \mathrm{mg} / \mathrm{ml})$, and Vancomycin $(0.5 \mathrm{mg} / \mathrm{mL})$ added to the sterile drinking water of mice as previously described [5]. After four weeks, Abx treatment was stopped and the mice were treatment with AOM and DSS combined with ETBF $\left(1 \times 10^{8}\right.$ colony forming units $)$ or Vehicle (E. coli MG1655 or the same volume of phosphate buffer saline) every day for the development of neoplastic lesions as previously described. ETBF was grown under anaerobic conditions at $37^{\circ} \mathrm{C}$ overnight prior to administration to mice essentially as described [29]. Signs of illness were monitored daily and body weight was recorded weekly.

\section{Microbial analysis of mouse stool}

Feces of all mice in the NS and YYFZBJS group were collected for gut microbiota analyses. Briefly, (i) genomic DNA was extracted using a Power Soil DNA Isolation Kit (MO BIO Laboratories, Carlsbad, CA); (ii) the $16 \mathrm{~S}$ rDNA V4 region was amplified using the 515F and 806R primers; (iii) PCR product quantification, qualification, and purification were performed; (iv) library preparation and sequencing were performed on the MiSeq platform (Illumina, Inc, San Diego, CA). The 16S rRNA sequencing data were quality filtered using FLASH (Fast Length Adjustment of Short reads, Version 1.2.11). Operational taxonomic units (OTUs) were picked at a $97 \%$ sequence similarity cut-off, and the purified amplicons were sequenced on an Illumina MiSeq platform at Majorbio Bio-pharm Technology Co. Ltd according to the standard protocols.

\section{Histology and Immunohistochemistry}

The whole intestine was removed immediately after sacrifice and opened longitudinally after washed with ice-cold phosphate buffer saline (PBS) as previously described [5]. The number, location, and size of visible tumors throughout the intestine were measured to calculate the incidence of adenoma. Tumor numbers were counted and grouped based on sizes: $<3 \mathrm{~mm}, 3-5 \mathrm{~mm}$ and $>5 \mathrm{~mm}$. Tissue sections were fixed in $10 \%$ formalin followed by paraffin embedding. Then they were stained with hematoxylin and eosin for pathological evaluation by a pathologist blinded to the experimental groups. Histological analysis for polyp, adenoma, and adenocarcinoma was performed by a board-certified pathologist (PV) as previously described [5]. The histology scoring criteria is as follows: $0=$ normal, $1=$ moderate, $2=$ marked and $3=$ severe.

For the murine samples, immunohistochemistry was performed to detect total Ki67 (anti-mouse Ki67, Abcam), PCNA (anti-mouse PCNA, Abcam); all stains used horseradish peroxidase-conjugated antibody, with chromogenic detection with the substrate 3-3'-diaminobenzidine, and finally counterstained with hematoxylin.

\section{Cytokine antibody arrays}


Serum samples were screened in duplicates using a Mouse Cytokine Array QAM-INF-1 (RayBiotech) containing slides coated with 40 different cytokines according to the manufacturer's guidelines with some modifications as previously described [5]. Briefly, the arrays were blocked, incubated with $100 \mathrm{~mL}$ of condition medium overnight, followed by biotin-conjugated antibodies (1/250) incubation for $2 \mathrm{hr}$ and with HRP-linked secondary antibody $(1 / 1000)$ for $1 \mathrm{hr}$. The membranes were incubated with a peroxidase substrate, and the results were documented using XAR films. Quantitative array analysis was performed using Array Vision Evaluation 8.0 (GE Healthcare Life Science).

\section{Co-incubation of ETBF and CRC cells}

ETBF were grown in Luria-Bertani (LB) broth at $37^{\circ} \mathrm{C}$ overnight to mid-log phase. Concentration of bacteria was adjusted based on the optical density reading at $600 \mathrm{~nm}$ analyzed by NanoDrop ND-2000. Bacteria were diluted to 1:10 with appropriate medium prior to co-culture with macrophages and CRC cells [30]. Briefly, MC-38 cells w/o treatment of IL-4-primed macrophage RAW264.7 cells were seeded into 6-well lumox plates (Vivascience, Gloucestershire, UK) and grown to $70 \%$ confluence before co-cultured with ETBF at $10^{6} \mathrm{CFU} / \mathrm{ml}$ in RPMI-1640 supplemented with $10 \%$ fetal bovine serum for $6 \mathrm{~h}$. ETBF were allowed to infect the monolayers of CRC cells. All extracellular bacteria were killed by gentamicin (500 $\mathrm{\mu g} / \mathrm{ml}$ for $20 \mathrm{~min})$ and the dead bacteria were removed by extensive washing with PBS [30].

\section{RNA-seq and analysis}

Total RNAs from CRC cells were isolated with trizol and the RNA-seq library was prepared by the Icsciences Co. Ltd. RNA-Seq FASTQ files were processed using the RNA-Seq module implemented in the CLC Genomics Workbench v8.0 software (Qiagen Bioinformatics) with default settings as previously reported [31]. Reads counts were normalized based on RPKM (Fragments Per Kilobase per Million mapped reads). Heatmaps were drawn using multiple array viewer.

\section{Western blot and Immunofluorescence analysis}

Whole cell lysates were prepared for the Western blot analysis of Arg-1, iNOS, p-JNK, p-STAT3, p-ERK, and GAPDH expression as previously reported [32] Briefly, the cells were maintained on ice in lysis buffer for $2 \mathrm{~h}$ before being collected with a cell scraper. The sample was centrifuged, and the supernatant was collected and stored at $-80^{\circ} \mathrm{C}$ until use. A densitometric analysis was performed using the Scion Imaging application (Scion Corporation), and GAPDH was used as an internal reference.

Immunofluorescence analyses in RAW264.7 cells were incubated with rabbit anti- Arg-1 primary antibody (Bioss Antibodies) prepared in blocking solution (10\% horse serum, $0.1 \%$ Triton X-100, $0.02 \%$ sodium azide, PBS) followed by incubation with a fluorescent dye-conjugated donkey anti-rabbit secondary antibody (Molecular Probes). DAPI (4=,6-diamidino-2-phenylindole) (Life Technologies) staining was performed following the manufacturer's protocol, and fluorescence images were obtained using a Diaphot 300 fluorescence microscope (Nikon) with SPOT software (Sterling Heights) as previously described [33].

\section{Flow Cytometry}


A phenotype analysis of the macrophages was performed with a BD FACS Ariall flow cytometer (BD, USA) as previously described [16]. Briefly, the cells were labeled with CD11b- FITC, F4/80-PE, and CD206-APC (eBioscience, San Diego, CA) following the manufacturer's protocol. To analyze the prevalence of M2 macrophages, the cells were incubated with PE-conjugated F4/80 antibodies or APC-conjugated CD206. Flow cytometry was performed using a FACS CaliburTM flow cytometer, and the data were analyzed using Paint-A-Gate software (Becton Dickinson).

\section{Analysis of cytokine expression in mouse tumor}

The mouse tumor samples were analyzed to detect mouse cytokines by ELISA according to the manufacturer's instructions (eBioscience) and as previously described [5].

\section{Clonogenic assays}

Inoculated MC-38 cells in a six-well plate (approximately 100 cells/well) were cultured in complete medium with different groups of supernatants for 2 days. Then, the cells were washed twice with PBS, cultured in normal medium and allowed to form colonies for 14 days. The medium was replaced every four to five days. The colonies were washed three times with PBS. The, the colonies were fixated with $95 \%$ ethanol for twenty minutes and stained with $1 \%$ crystal violet.

\section{Transwell co-culture assay}

MC-38 cells and BMDMs were co-cultured with BMDMs ETBF for $6 \mathrm{~h}$ as described in Co-incubation of Methods. For the cell proliferation assay, the inserts were placed in a 24-well plate with pre-seeded TAMs. Then, cancer cell activity was detected by the trypan blue method. For the cell invasion ability assay, TAMs were seeded on the bottom of a 24-well plate with cancer cells in a Transwell chamber such that both cell populations were exposed to the same conditions.

\section{Results}

\section{YYFZBJS suppresses colorectal tumorigenesis in the CAC mouse model}

Previously, we showed that YYFZBJS were sufficient to inhibit intestinal tumorigenesis in $\mathrm{Apc}^{\mathrm{Min} /+}$ mice. In the present study, to investigate the preventive effects of YYFZBJS on CAC formation, the mouse treatment was initiated at the age of 6 weeks. The structures of the determined experiment from the herbs are shown in Fig. 1A. After the AOM/DSS feeding, a significant body weight loss (Fig. 1B), which has been weakened by YYFZBJS in a dose-dependent manner.

In addition, YYFZBJS decreased the colon adenoma incidence and tumor number with $7.89 \%, 19.74 \%$ and $53.95 \%$ respectively in a dose-dependent manner (Fig. 1C\&E). As shown in Fig. 1D and Supplementary Table S1, compared to the AOM/DSS-treated group, the mice treated with YYFZBJS or Aspirin showed body weight recovery. Figure 1F shows that the administration of YYFZBJS significantly 
increased the survival rate of the mice compared with that of the mice in the NS group (Control), which received an equivalent volume $(\mu \mathrm{L})$ of normal saline. Notably, the numbers of polyps in all three YYFZBJS groups were all much fewer than that of the non-treated control group (Fig. 1G). These results suggest that YYFZBJS is effective in protecting against AOM/DSS-induced colitis-associated tumorigenesis.

\section{YYFZBJS modulates the gut microbiome composition in the CAC mouse model}

To investigate if colon protective effect of YYFZBJS is, at least in part, we documented 16S rRNA sequencing to detect the influence of YYFZBJS on microbiota composition in AOM-initiated and DSSpromoted colon carcinogenesis model. First of all, we compared the gut microbiota between YYFZBJS and Control group through linear discriminant analysis effect size (LEfSe) to identify the specific microbiota linked to the colon carcinogenesis model. Dubosiella, Clostridium and Bacillus, whose members are generally associated with butyrate production, were more abundant in the YYFZBJS group (Fig. 2A). Both Chao and Shannon index did not change significantly in four groups, indicating that YYFZBJS treatment could not alter OTU number of gut microbiota (Fig. 2B). However, the Simpson indices between YYFZBJS and the control group were statistically significant, which suggested that YYFZBJS stronger positive effects on the a diversity of the gut microbiota (Fig. 2B). The gut microbiota of all the samples was dominated by four major phyla: Bacteroidetes, Ruminococcaceae, Lactobacillus, and Muribaculaceae (data not shown). The genus-level analysis revealed that YYFZBJS increased the relative abundance of pathogenic bacteria, such as Lactobacillus, Ruminococcaceae, and Clostridium (Fig. 2C\&D). Notably, compared with the control, a lower mRNA expression of Enterotoxigenic bacteroides fragilis (ETBF) and Akkermansia were observed in different dose of YYFZBJS treatment mice (Fig. 2E). Additionally, a relatively upregulation of Lactobacillus rhamnosus (LGG) and clostridium butyricum was observed in mice fed with YYFZBJS compared with that of the control, and this effect was shown as a dose-dependent manner (Fig. 2E and Supplementary Table S2).

\section{ETBF infection enhances colonic tumorigenesis in AOM/DSS model}

Previously, we confirmed the effect of YYFZBJS on inhibition of tumor development in AOM/DSS mice. A large number of studies have confirmed that ETBF can accelerate tumor occurrence and malignant. In order to investigate the mechanism of ETBF in carcinoma arising, germ-free (Abx) mice with AOM and DSS was used as a germ-free (GF) mode. The experimental design and timeline are shown in Fig. 3A. Consistent with the previous results, no difference was noted in the length of colon among the treatment groups during the experiment (Fig. 3B), but the numbers of polyps in ETBF groups were all much more than those in the non-treated control group (Fig. 3C). Similarly, compared to the AOM/DSS-treated group, ETBF increased the colon adenoma incidence and tumor number by $12 \%$ and $31.58 \%$, respectively (Fig. 3D, E). Since Ki67, PCNA are cell proliferation markers, we examined the localization and expression levels of Ki67 and PCNA by immunohistochemistry in the tumors from the AOM/DSS mice with or without the ETBF treatment (Fig. 3F). 
Emerging studies have found that the promotion effect of ETBF in development of tumorigenesis is mostly related to tumor microenvironment $[2,9]$. To investigate the mechanism of ETBF in colorectal tumorigenesis, we adopted a cytokine antibody array (Ray Biotech), which demonstrated that compared to the Vehicle group, the ETBF treated mice secrete lower levels of inflammatory cytokines/chemokines, including IL-4, IL-13, TNF-a and so on (Fig. 3G). We found that ETBF strains dramatically increased protein expression levels of M2 (IL-4, IL-10, IL-13, etc.) and decreased the levels of ICAM-1, IL-1 $\beta$ and so on, which representative $\mathrm{M} 2$ macrophages (Fig. $3 \mathrm{H}$ ).

\section{ETBF infection enhances colonic tumorigenesis in AOM/DSS model}

To determine whether the presence of gut commensal bacteria affects regulatory CRC cells and macrophage in vitro, ETBF was co-incubated with MC-38 cells with RAW264.7 macrophages in a Transwell system in which the macrophages were located in the chamber, and the cancer cells were located in the cell plate (Fig. 4A). The heatmap displays relative fold changes in expression levels of proliferation markers of cancer cell and stem cell in MC-38 cells, macrophages co-cluture with MC-38 cells, and ETBF incubated macrophages with MC-38 cells (Fig. 4B).

To explore underlying mechanisms of these ETBF-macrophages phenomena on CRC cells, we placed MC38 cells in a Transwell system co-cultured with RAW264.7 cells for $48 \mathrm{~h}$ to observe the mechanism of ETBF-CRC cell in the macrophages (Fig. 4C). Interestingly, he changes in protein expression of JNK, STAT3 and NFK-B were up-regulation in ETBF incubated- macrophages and CRC cells compared with that not expose in ETBF system (Fig. 4D). Consistent with the results of western blot, the effect of ETBF on M2 polarization is verified by the increase of Arg-1 aggregation in IHC analysis (Fig. 4E).

\section{YYFZBJS inhibited ETBF-induced colorectal tumor development in AOM/DSS model}

To further investigate if regulation of microbiota composition contributed to protective effect of ETBF, we co-housed the GF/AOM/DSS-treated mice which received ETBF or ETBF combined YYFZBJS to test if the protection effect of YYFZBJS could be counteracted by gut microbiota transmission. We conducted the experiment as shown in Supplementary Fig. S1. We found that ETBF combined with YYFZBJS dramatically decreased polyp numbers in the GF/AOM/DSS model compared to mice given only ETBF strains (Fig. 5A and B). The result of colon length still did not change significantly with or without YYFZBJS, indicating that YYFZBJS treatment could not alter colon length of GF/AOM/DSS mice infected with ETBF (Fig. 5B). However, in accord with the result in vitro, the expression levels of the STAT3 were greatly decreased in the ETBF combined with YYFZBJS group compared with those in the ETBF group (Fig. 5C). Furthermore, the ETBF combined with YYFZBJS -treated GF/AOM/DSS mice demonstrated decreased CD163 and Arg-1 immunoreactivity compared with the ETBF (Fig. 5D). Remarkably, after the ETBF combined with YYFZBJS feeding, the GF/AOM/DSS mice showed a significant change in M2 polarization, especially a reduction in CD206 in the tumor tissue (Fig. 5E). These data support the role of ETBF combined with YYFZBJS in the process of inflammation during intestinal tumorigenesis. IL-12, 
TNF- $\alpha$ and IL-6 are responsible for inducing the M1 phenotype, while IL-4, IL-10 and TGF- $\beta$ are M2polarizing cytokines. As a result, the secretion levels of the M1-related gene expression of IL-12 and TNF-a were significantly higher and the IL-10 and TGF- $\beta$ levels were significantly lower in the colon of the ETBF combined with YYFZBJS mice compared with those in the GF/AOM/DSS mice treated with ETBF only (Fig. 5F).

\section{YYFZBJS inhibited tumor cell proliferation through regulating ETBF primed BMDMs in vitro}

To determine whether the presence of YYFZBJS affects macrophages in vitro, MC-38 cells were coincubated with M2 macrophages which isolated from the BMDMs of YYFZBJS group mice (Fig. 6A). The result of Fig. 6B displayed significantly lower colony-forming unit per milliliter compared to controls when CRC cells incubated with YYFZBJS-primed BMDMs. To explore underlying mechanisms of these YYFZBJS-primed macrophages phenomena on CRC cells, we determined the targets of significant differential expression in the previous experiment, and found a significant decrease in c-Met, MMPs and cyclinD1 expression in MC-38 cells (Fig. 6C). Similarly, the YYFZBJS-primed BMDMs inhibited the infiltration ability of the tumor cells (Fig. 6D). Based on the RNA sequencing in previous CRC cell results, the noticeable changes of mRNA were analyzed by PCR in MC-38 cells co-cluture with YYFZBJS-primed BMDMs (Fig. 6E). YYFZBJS primed BMDMs could significantly decrease the phosphorylation of STAT3, while BMDMs from C57 mice could effectively increase the level of STAT3 and p- STAT3 (Fig. 6F).

\section{Discussion}

Epidemiologic studies suggest that herbal medicines reduce colon cancer risk in humans [34]. Previous studies have already revealed the anti-tumor effect of YYFZBJS, and its modulating effect in gut microbiome as an important role for delaying the progression of colonic tumorigenesis [5]. Several recent studies have confirmed the link between dysbiosis of the gut microbiome and colorectal cancer [35, 36]. Our previous findings have confirmed that, ETBF, one of the most regulated monomers by YYFZBJS, promoted the proliferation of tumor cells through immunity [5]. However, no studies investigating the impact of TCM on the balance between gut microbiome and immune cell in the colonic tumorigenesis have been reported.

Here, we showed that YYFZBJS, a traditional Chinese herbal medicine from Synopsis of Golden Chamber, significantly reduced tumor multiplicity and numbers in the AOM/DSS mouse model. This is in lines with previous studies where happen in $A p c^{M i n /+}$ mice; YYFZBJS FMT administration modulates microbial consortia on colorectal carcinogenesis and results in a significant reduction in overall polyp number and size. In the present study, OTUs results further showed that the bacteria of Bacteroidetes, Ruminococcaceae, and Lactobacillus, might play an active role in both pro- and anti-inflammatory macrophages regulatory pathways. Interestingly, several studies have highlighted the inducement of colon macrophages is closely related to the gut microbiome and to play roles in promoting tumor function $[37,38]$. Reports showed that the intestinal flora activate mucosal macrophages through 
intestinal mucosal $\mathrm{M}$ cells, triggering innate immune responses and producing marginal effects, thereby promoting the occurrence and development of CRC [39]. As expected, our data also found that ETBF administration modulates microbial consortia on colorectal carcinogenesis and results in a significant increase in overall polyp number and size. Further study confirmed that ETBF promotes the M2 polarization of macrophages to help the growth of tumor cells, resulting in promoting the occurrence and development of CRC. Ours and others data have shown that M2 macrophages promote colorectal cancer metastasis via M2 macrophage-secreted protein and/or regulatory factors $[16,40]$.

To our knowledge, the enterotoxigenic $B$. fragilis (ETBF) forms biofilm and produce toxin and play a role in $\mathrm{CRC}$, whereas the non-toxigenic B. fragilis (NTBF) does not produce toxin. ETBF is associated with IBD due to the abnormal regulation of immune response to bacteria [41]. Pathogenic bacteria are capable of stimulating infection, inflammation and carcinogenesis, whereas the relationship between IBD and CRC is well established [42]. However, in ETBF-induced carcinogenesis, the role of Treg cells is to promote cancer $[43,44]$ A previous study showed Treg cells limit ETBF-induced colitis but promote the expansion of Th17 cells and the overexpression of tumor IL-17 by limiting the availability of IL-2 in the tumor environment [44]. ETBF infection-mediated tumorigenesis is contributed by STAT3 signaling of Th17 cells or colon epithelial cells in APCMin/+ mice [45]. Specifically, ETBF has the ability to activate STAT3 rapidly in both colonic epithelial cells and colonic mucosal immune cells through phosphorylation and nuclear translocation. Similar to our in vitro results, our microarray data suggest that ETBF-primed macrophage evoke the activation of tumor cell proliferation proteins and oncogenic pathways in CRC carcinogenesis, especially on STAT3, JNK, and NF-KB signaling because of significant impacts on M2 macrophage polarization.

Accumulating data also indicated that the percentage of M2 macrophage is inversely related to increasing the risk for the progression of cancer $[46,47]$. For CRC patients, increased numbers of M2 macrophage had been found in peripheral blood, tumor-draining lymph node (DLN), and tumor microenvironment [48]. Coincidently, M2 macrophage have also been reported by clinical observations and mechanistic studies, to play an indispensable role as a promoter of tumor growth because of its suppressive effects on the autologous effector T-cell responses. In our previous study, we noted that M2 phenotype macrophages in particular seemed to expand in the polyps from the AOM/DSS mice by upregulating CD206, Arg-1, IL-10, and TGF- $\beta$, which are considered the main M2 markers [16]. In the current study, we also found that ETBF infection increases the expression of IL-6, IL-10, and TGF- $\beta$ in peripheral blood of AOM/DSS mice and Arg-1 expression in ETBF primed macrophages. It suggested that ETBF provided a tumor-initiating factor in M2- macrophage mediated tumorigenesis.

To explore the anti-tumor mechanism of YYFZBJS in the gut microbiome, it is the first time that the BMDMs from mice gavaged with YYFZBJS has been co-cultured with tumor cells in our study (Fig. 6A). Interestingly, YYFZBJS primed macrophages showed insignificant changes in the cell viability of CRC MC-38 cells in a time and dose-dependent manner. Our results also show that in supernatants of cultured tumor cells, the tumorigenic correlation genes, protein and phosphorylation of protein such as STAT3 in CRC cells was suppressed by the YYFZBJS primed macrophages, indicating that YYFZBJS inhibits the 
STAT3, JNK, and Nkb signaling pathways. Moreover, our results indicated that YYFZBJS indirectly inhibited colon cell proliferation and altered the differentiation of the M2 phenotype in the co-culture microenvironment, suggesting that the role of TAMs in promoting tumor growth can be reduced by YYFZBJS.

\section{Conclusions}

In summary, we demonstrated that YYFZBJS prevents colon tumorigenesis in AOM/DSS animal models. The anti-tumor effect was mediated by the ETBF for promoting macrophage polarization and leading to the phosphorylation of STAT3 and proliferation of tumor cells. Specifically, we demonstrate that growth of cancer cells can be influenced by the ETBF via BMDMs from the mice gavaged with YYFZBJS. These findings suggest potential new mechanisms by TCM prevent colitis-associated tumorigenesis by inhibiting commensal microbiota via M2 macrophage polarization. This discovery helps us better understand the anticancer effect of YYFZBJS and its ability to remodel the gut microbiota, leading to regulation of immunity and delay of carcinogenesis.

\section{Abbreviations}

CRC: Colorectal cancer; ETBF: Bacteroides fragilis; GF: germ free; YYFZBJS: Yi-Yi-Fu-Zi-Bai-Jiang-San; DAl: disease activity index; AOM: azoxymethane/dextran sulfate sodium: AOM/DSS; TAMs: tumorassociated macrophages; ACF: aberrant crypt foci; PCNA: Proliferating Cell Nuclear Antigen; IFN-ץ: interferon gamma; IL-4/6/10: interleukin-4/6/10; TNF-a: tumor necrosis factor- $\alpha$; Apc: adenomatous polyposis coli; PBS: Phosphate-buffered saline; TCM: Traditional Chinese Medicine; IHC: Immunohistochemistry; qRT-PCR: real time quantitative reverse transcription PCR;

\section{Declarations}

\section{Acknowledgments}

The authors thank Dr. Lu Zhang for the data from the analysis of fecal flora.

\section{Declarations}

\section{Authors' contributions}

HS and HRZ designed the experiments. YBX and YLC collected the data. WFS and YQY provided materials and technique supports. NC drafted the manuscript. LMY supervised the experiments. All authors have read and approved the final manuscript.

\section{Funding}

This research was supported by the National Natural Science Foundation of China (No. 81874399), the Project of Hospital Development Center in Shanghai (No. SHDC2020CR4047). 
Availability of data and materials

The datasets used or analyzed during the current study are available from the corresponding author on reasonable request.

\section{Ethical approval and consent to participate}

All animal protocols were approved by the Institutional Animal Use and Care Committee. All experiments and animal care procedures were approved by the Shanghai Medical Experimental Animal Care Commission and were conducted in accordance with the provisions and general recommendations of the Chinese Experimental Animals Administration Legislation. All animals were received humane care according to the National Institutes of Health (USA) guidelines.

\section{Consent for publication}

Not applicable.

\section{Competing interests}

The authors declare that they have no competing interests.

\section{References}

1. Lee YK, Mehrabian P, Boyajian S, Wu WL, Selicha J, Vonderfecht S, Mazmanian SK. The Protective Role of Bacteroides fragilis in a Murine Model of Colitis-Associated Colorectal Cancer. mSphere. 2018;3(6):e00587-18.

2. Hwang S, Jo M, Hong JE, Park CO, Lee CG, Rhee KJ. Protective Effects of Zerumbone on Colonic Tumorigenesis in Enterotoxigenic Bacteroides fragilis (ETBF)-Colonized AOM/DSS BALB/c Mice. Int J Mol Sci. 2020;21(3):857.

3. Gopalakrishnan V, Helmink BA, Spencer CN, Reuben A, Wargo JA. The Influence of the Gut Microbiome on Cancer, Immunity, and Cancer Immunotherapy. Cancer Cell. 2018;33(4):570-580.

4. Si H, Yang Q, Hu H, Ding C, Wang H, Lin X. Colorectal cancer occurrence and treatment based on changes in intestinal flora. Semin Cancer Biol. 2020:S1044-579X(20)30100-0.

5. Sui H, Zhang L, Gu K, Chai N, Ji Q, Zhou L, Wang Y, Ren J, Yang L, Zhang B, et al. YYFZBJS ameliorates colorectal cancer progression in $\mathrm{Apc}^{\mathrm{Min} /+}$ mice by remodeling gut microbiota and inhibiting regulatory T-cell generation. Cell Commun Signal. 2020;18(1):113.

6. Boleij A, Hechenbleikner EM, Goodwin AC, Badani R, Stein EM, Lazarev MG, Ellis B, Carroll KC, Albesiano $\mathrm{E}$, Wick EC, et al. The Bacteroides fragilis toxin gene is prevalent in the colon mucosa of colorectal cancer patients. Clin Infect Dis. 2015;60(2):208-15.

7. Sears CL, Islam S, Saha A, Arjumand M, Alam NH, Faruque AS, Salam MA, Shin J, Hecht D, Weintraub A, et al. Association of enterotoxigenic Bacteroides fragilis infection with inflammatory diarrhea. Clin 
Infect Dis. 2008;47(6):797-803.

8. Dejea CM, Fathi P, Craig JM, Boleij A, Taddese R, Geis AL, Wu X, DeStefano Shields CE, Hechenbleikner EM, Huso DL, et al. Patients with familial adenomatous polyposis harbor colonic biofilms containing tumorigenic bacteria. Science. 2018;359(6375):592-597.

9. Gu T, Li Q, Egilmez NK. IFNß-producing CX3CR1 ${ }^{+}$macrophages promote T-regulatory cell expansion and tumor growth in the APC ${ }^{\mathrm{min} /+} /$ Bacteroides fragilis colon cancer model. Oncoimmunology. 2019;8(12):e1665975.

10. Verma S, Prescott R, Cherayil BJ. The commensal bacterium Bacteroides fragilis down-regulates ferroportin expression and alters iron homeostasis in macrophages. J Leukoc Biol. 2019;106(5):1079-1088.

11. Geis AL, Fan H, Wu X, Wu S, Huso DL, Wolfe JL, Sears CL, Pardoll DM, Housseau F. Regulatory T-cell Response to Enterotoxigenic Bacteroides fragilis Colonization Triggers IL17-Dependent Colon Carcinogenesis. Cancer Discov. 2015;5(10):1098-109.

12. Gu T, De Jesus M, Gallagher HC, Burris TP, Egilmez NK. Oral IL-10 suppresses colon carcinogenesis via elimination of pathogenicCD $4^{+}$T-cells and induction of antitumor CD8 ${ }^{+}$T-cell activity. Oncoimmunology. 2017;6(6):e1319027.

13. Kwong LN, Dove WF. APC and its modifiers in colon cancer. Adv Exp Med Biol. 2009;656:85-106.

14. Takahashi M, Wakabayashi K. Gene mutations and altered gene expression in azoxymethaneinduced colon carcinogenesis in rodents. Cancer Sci. 2004;95(6):475-80.

15. DeNardo DG, Ruffell B. Macrophages as regulators of tumour immunity and immunotherapy. Nat Rev Immunol. 2019;19(6):369-382.

16. Sui H, Tan H, Fu J, Song Q, Jia R, Han L, Lv Y, Zhang H, Zheng D, Dong L, et al. The active fraction of Garcinia yunnanensis suppresses the progression of colorectal carcinoma by interfering with tumorassociated macrophage-associated M2 macrophage polarization in vivo and in vitro. FASEB J. 2020;34(6):7387-7403.

17. Yahaya MAF, Lila MAM, Ismail S, Zainol M, Afizan NARNM. Tumour-Associated Macrophages (TAMs) in Colon Cancer and How to Reeducate Them. J Immunol Res. 2019;2019:2368249.

18. Tan SY, Fan Y, Luo HS, Shen ZX, Guo Y, Zhao LJ. Prognostic significance of cell infiltrations of immunosurveillance in colorectal cancer. World J Gastroenterol. 2005;11(8):1210-4.

19. Cui YL, Li HK, Zhou HY, Zhang T, Li Q. Correlations of tumor-associated macrophage subtypes with liver metastases of colorectal cancer. Asian Pac J Cancer Prev. 2013;14(2):1003-7.

20. Jayme TS, Leung G, Wang A, Workentine ML, Rajeev S, Shute A, Callejas BE, Mancini N, Beck PL, Panaccione $\mathrm{R}$, et al. Human interleukin-4-treated regulatory macrophages promote epithelial wound healing and reduce colitis in a mouse model. Sci Adv. 2020;6(23):eaba4376.

21. Tilg H, Adolph TE, Gerner RR, Moschen AR. The Intestinal Microbiota in Colorectal Cancer. Cancer Cell. 2018;33(6):954-964. 
22. Dumitrescu O, Choudhury P, Boisset S, Badiou C, Bes M, Benito Y, Wolz C, Vandenesch F, Etienne J, Cheung $\mathrm{AL}$, et al. Beta-lactams interfering with PBP1 induce Panton-Valentine leukocidin expression by triggering sarA and rot global regulators of Staphylococcus aureus. Antimicrob Agents Chemother. 2011;55(7):3261-71.

23. Fessler J, Matson V, Gajewski TF. Exploring the emerging role of the microbiome in cancer immunotherapy. J Immunother Cancer. 2019;7(1):108.

24. Missiaglia E, Jacobs B, D'Ario G, Di Narzo AF, Soneson C, Budinska E, Popovici V, Vecchione L, Gerster $S$, Yan P, et al. Distal and proximal colon cancers differ in terms of molecular, pathological, and clinical features. Ann Oncol. 2014;25(10):1995-2001.

25. van Leeuwen BL, PåhIman L, Gunnarsson U, Sjövall A, Martling A. The effect of age and gender on outcome after treatment for colon carcinoma. A population-based study in the Uppsala and Stockholm region. Crit Rev Oncol Hematol. 2008;67(3):229-36.

26. Zheng $Y Y$, Viswanathan B, Kesarwani P, Mehrotra S. Dietary agents in cancer prevention: an immunological perspective. Photochem Photobiol. 2012;88(5):1083-98.

27. Ying W, Riopel M, Bandyopadhyay G, Dong Y, Birmingham A, Seo JB, Ofrecio JM, Wollam J, Hernandez-Carretero A, Fu W, et al. Adipose Tissue Macrophage-Derived Exosomal miRNAs Can Modulate In Vivo and In Vitro Insulin Sensitivity. Cell. 2017;171(2):372-384.e12.

28. Sui H, Liu X, Jin BH, Pan SF, Zhou LH, Yu NA, Wu J, Cai JF, Fan ZZ, Zhu HR, et al. Zuo Jin Wan, a Traditional Chinese Herbal Formula, Reverses P-gp-Mediated MDR In Vitro and In Vivo. Evid Based Complement Alternat Med. 2013;2013:957078.

29. Hwang S, Jo M, Hong JE, Park CO, Lee CG, Yun M, Rhee KJ. Zerumbone Suppresses Enterotoxigenic Bacteroides fragilisInfection-Induced Colonic Inflammation through Inhibition of NFKB. Int J Mol Sci. 2019;20(18):4560.

30. Li R, Zhou R, Wang H, Li W, Pan M, Yao X, Zhan W, Yang S, Xu L, Ding Y, et al. Gut microbiotastimulated cathepsin $\mathrm{K}$ secretion mediates TLR4-dependent M2 macrophage polarization and promotes tumor metastasis in colorectal cancer. Cell Death Differ. 2019;26(11):2447-2463.

31. Jaeckel S, Kaller M, Jackstadt R, Götz U, Müller S, Boos S, Horst D, Jung P, Hermeking H. Ap4 is rate limiting for intestinal tumor formation by controlling the homeostasis of intestinal stem cells. Nat Commun. 2018;9(1):3573.

32. Sui H, Xu H, Ji Q, Liu X, Zhou L, Song H, Zhou X, Xu Y, Chen Z, Cai J, et al. 5-hydroxytryptamine receptor (5-HT1DR) promotes colorectal cancer metastasis by regulating Axin1/ $\beta$-catenin/MMP-7 signaling pathway. Oncotarget. 2015;6(28):25975-87.

33. Sui H, Zhao J, Zhou L, Wen H, Deng W, Li C, Ji Q, Liu X, Feng Y, Chai N, et al. Tanshinone IIA inhibits $\beta$ catenin/VEGF-mediated angiogenesis by targeting TGF- $\beta 1$ in normoxic and HIF-1a in hypoxic microenvironments in human colorectal cancer. Cancer Lett. 2017;403:86-97.

34. Ji Q, Zhou L, Sui H, Yang L, Wu X, Song Q, Jia R, Li R, Sun J, Wang Z, et al. Primary tumors release ITGBL1-rich extracellular vesicles to promote distal metastatic tumor growth through fibroblast-niche formation. Nat Commun. 2020;11(1):1211. 
35. Long X, Wong CC, Tong L, Chu ESH, Ho Szeto C, Go MYY, Coker OO, Chan AWH, Chan FKL, Sung JJY, et al. Peptostreptococcus anaerobius promotes colorectal carcinogenesis and modulates tumour immunity. Nat Microbiol. 2019;4(12):2319-2330.

36. Li H, Xu F, Li S, Zhong A, Meng X, Lai M. The tumor microenvironment: An irreplaceable element of tumor budding and epithelial-mesenchymal transition-mediated cancer metastasis. Cell Adh Migr. 2016;10(4):434-46.

37. Colby JK, Jaoude J, Liu F, Shureiqi I. Oxygenated lipid signaling in tumor-associated macrophagesfocus on colon cancer. Cancer Metastasis Rev. 2018;37(2-3):289-315.

38. Kay C, Wang R, Kirkby M, Man SM. Molecular mechanisms activating the NAIP-NLRC4 inflammasome: Implications in infectious disease, autoinflammation, and cancer. Immunol Rev. 2020;297(1):67-82.

39. Grivennikov SI, Wang K, Mucida D, Stewart CA, Schnabl B, Jauch D, Taniguchi K, Yu GY, Osterreicher $\mathrm{CH}$, Hung KE, et al. Adenoma-linked barrier defects and microbial products drive IL-23/IL-17-mediated tumour growth. Nature. 2012;491(7423):254-8.

40. Ha CWY, Martin A, Sepich-Poore GD, Shi B, Wang Y, Gouin K, Humphrey G, Sanders K, Ratnayake Y, Chan KSL, et al. Translocation of Viable Gut Microbiota to Mesenteric Adipose Drives Formation of Creeping Fat in Humans. Cell. 2020;183(3):666-683.e17.

41. Cheng WT, Kantilal HK, Davamani F. The Mechanism of Bacteroides fragilis Toxin Contributes to Colon Cancer Formation. Malays J Med Sci. 2020;27(4):9-21.

42. Cho M, Carter J, Harari S, Pei Z. The interrelationships of the gut microbiome and inflammation in colorectal carcinogenesis. Clin Lab Med. 2014;34(4):699-710.

43. Irrazabal T, Martin A. T Regulatory Cells Gone Bad: An Oncogenic Immune Response against Enterotoxigenic B. fragilis Infection Leads to Colon Cancer. Cancer Discov. 2015;5(10):1021-3.

44. Geis AL, Housseau F. Procarcinogenic regulatory T cells in microbial-induced colon cancer. Oncoimmunology. 2015;5(4):e1118601.

45. Chung L, Thiele Orberg E, Geis AL, Chan JL, Fu K, DeStefano Shields CE, Dejea CM, Fathi P, Chen J, Finard BB, et al. Bacteroides fragilis Toxin Coordinates a Pro-carcinogenic Inflammatory Cascade via Targeting of Colonic Epithelial Cells. Cell Host Microbe. 2018;23(2):203-214.e5.

46. Yang Y, Li L, Xu C, Wang Y, Wang Z, Chen M, Jiang Z, Pan J, Yang C, Li X, et al. Cross-talk between the gut microbiota and monocyte-like macrophages mediates an inflammatory response to promote colitis-associated tumourigenesis. Gut. 2020:gutjnl-2020-320777.

47. Xia Y, Rao L, Yao H, Wang Z, Ning P, Chen X. Engineering Macrophages for Cancer Immunotherapy and Drug Delivery. Adv Mater. 2020;32(40):e2002054.

48. Aalipour A, Chuang HY, Murty S, D'Souza AL, Park SM, Gulati GS, Patel CB, Beinat C, Simonetta F, Martinić I, et al. Engineered immune cells as highly sensitive cancer diagnostics. Nat Biotechnol. 2019;37(5):531-539.

\section{Figures}


Figure 1

A
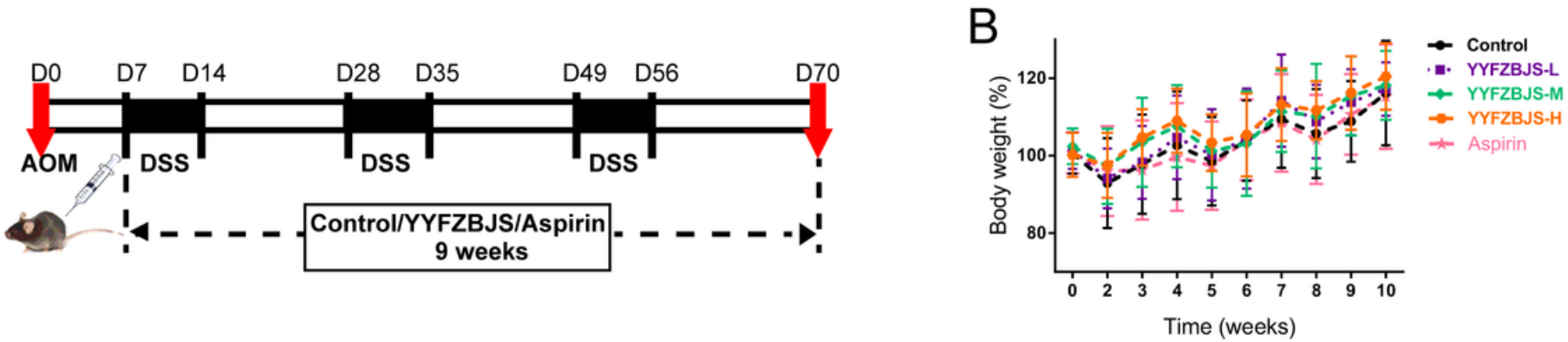

C

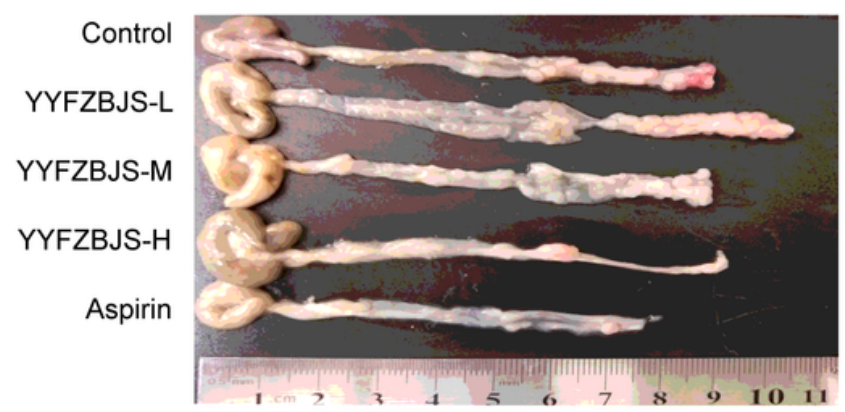

D

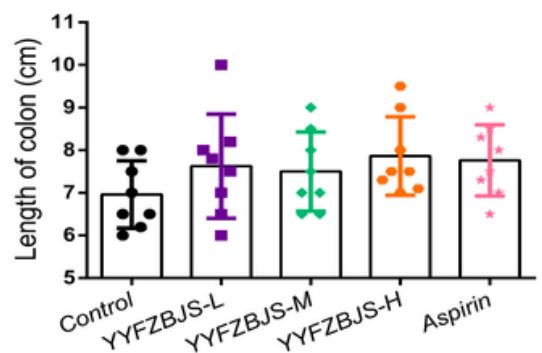

$E$

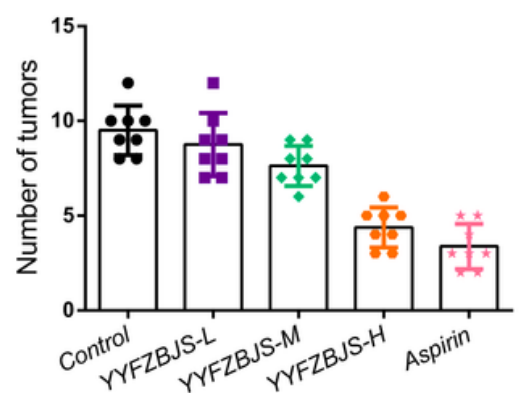

$\mathrm{F}$

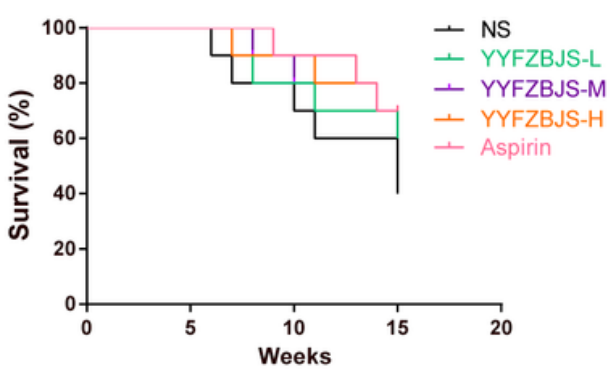

G

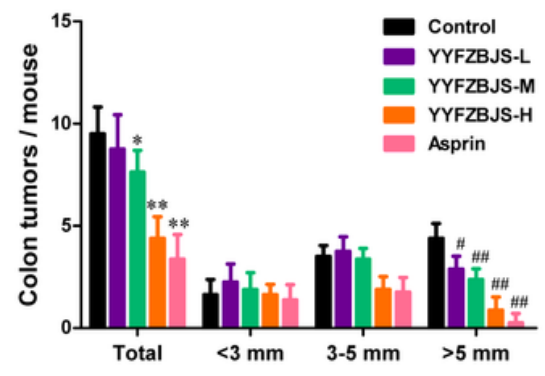

Figure 1

Effect of YYFZBJS on the occurrence and progression of CAC. (A) Experimental protocol used for the induction of the CAC model in C57BL/6J mice. (B, D, E, F) Effects of the model and treatment on mice body weight (B), colon length (D), number of intestinal polyps $(E)$ and the survival $(F)$ in mice. (C) Microscopic view of the colon in mice. $(G)$ The tumor size distribution in the intestine was listed and compared with control. Data are given as means \pm SD of 8 animals per experimental group, with Welch's correction, one-tailed t-test. \#P $<0.05$, \#\#P $<0.01$; ${ }^{*} \mathrm{P}<0.05$, $* \star \mathrm{P}<0.01$ vs. control. 
Figure 2
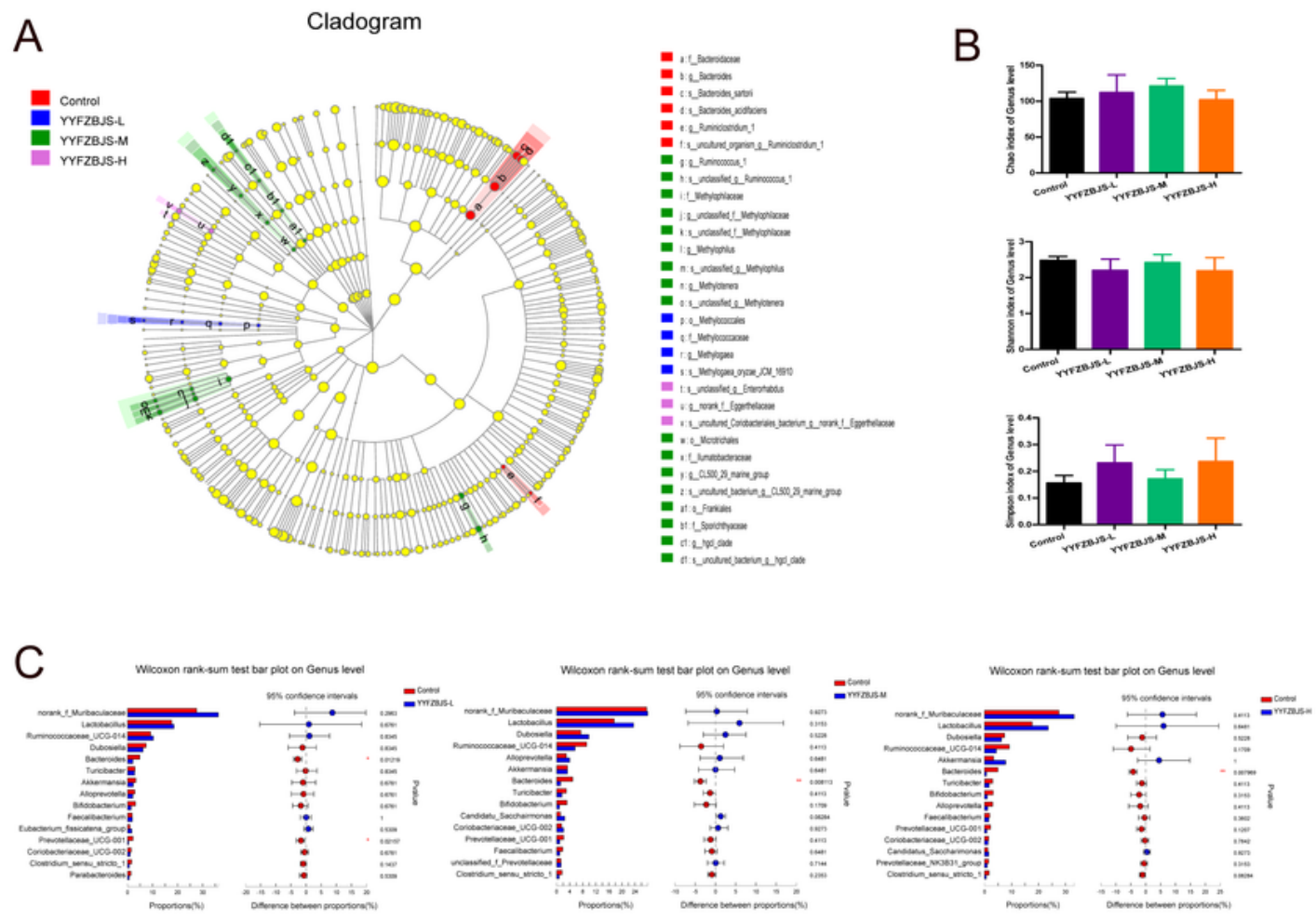

D

Community heatmap analysis on Genus level

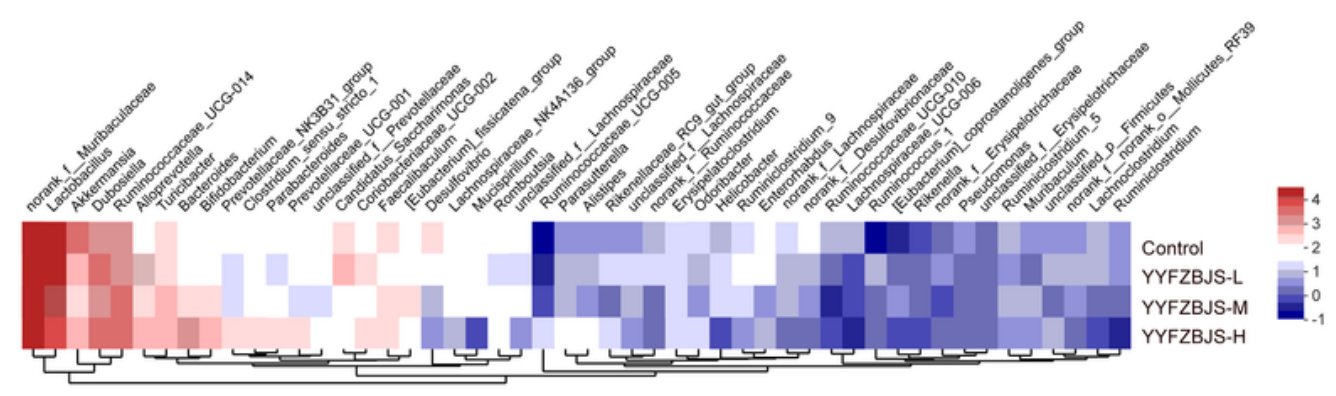

E
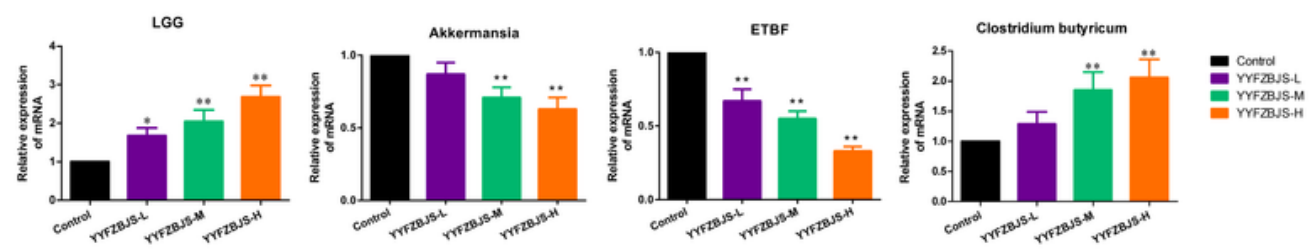

\section{Figure 2}

YYFZBJS modulated the composition of the gut microbiota in AOM/DSS mice. (A) Cladogram generated from the linear discriminant analysis effect size (LEfSe) between YYFZBJS group and Control group ( $n=8$ for each group). The analyses were performed at the end of the experiment. (B) a Diversity of gut microbiota for mice in four different groups. (C) Bar plot of compositional differences at the genus level in the gut microbiome of mice in the combination YYFZBJS group vs. the control group by the Wilcoxon 
rank-sum test. Data are expressed as mean $\pm S D$. * $0.01<P \leq 0.05 \nabla^{\star \star} 0.001<P \leq 0.01 \rrbracket^{\star \star \star *} P \leq 0.001$, Two-sided Hypotheses. (D) Heat map of Genus with relative abundances that are significantly different from their relative abundances at the time of YYFZBJS administration. The differentially enriched bacterial Genus in C57BL/6J mice receiving N.S and YYFZBJS. The relative abundance between control and treatment mice for the genus was calculated for each time. (E) Gut microbiota level was analyzed for mRNA expression of Lactobacillus rhamnosus (LGG), Akkermansia, ETBF, and Clostridium butyricum by quantitative RT-PCR. Quantifications of migrated cells from three independent experiments are shown as the mean \pm S.D. ${ }^{*} P<0.05,{ }^{*} \mathrm{P}<0.01$, vs. Control. 


\section{Figure 3}

A

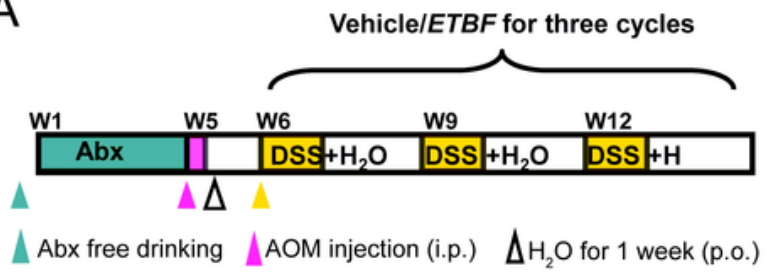

$\Delta$ DSs for 1 week, $\mathrm{H}_{2} \mathrm{O}$ for 2 weeks (p.o.)
B

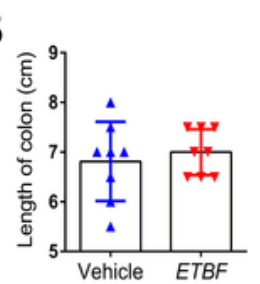

C

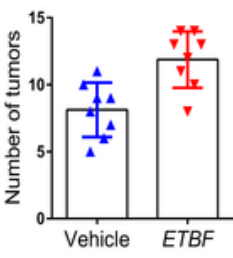

$\mathrm{D}$

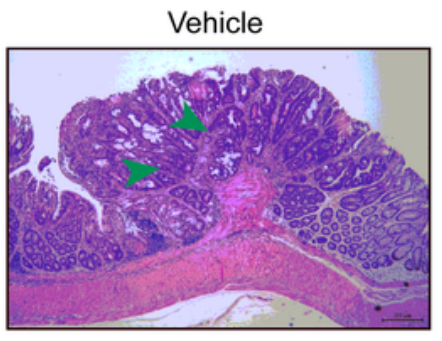

ETBF

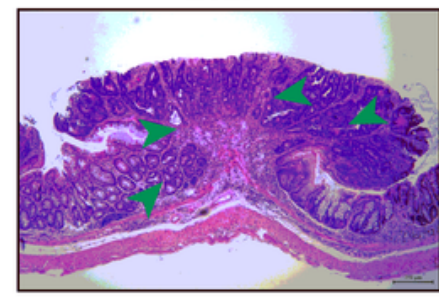

$\mathrm{E}$

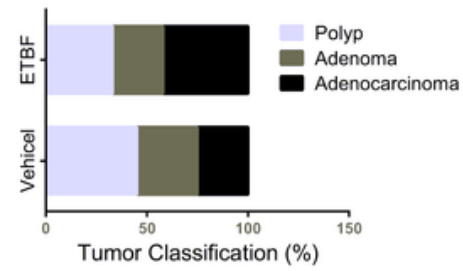

$\mathrm{F}$
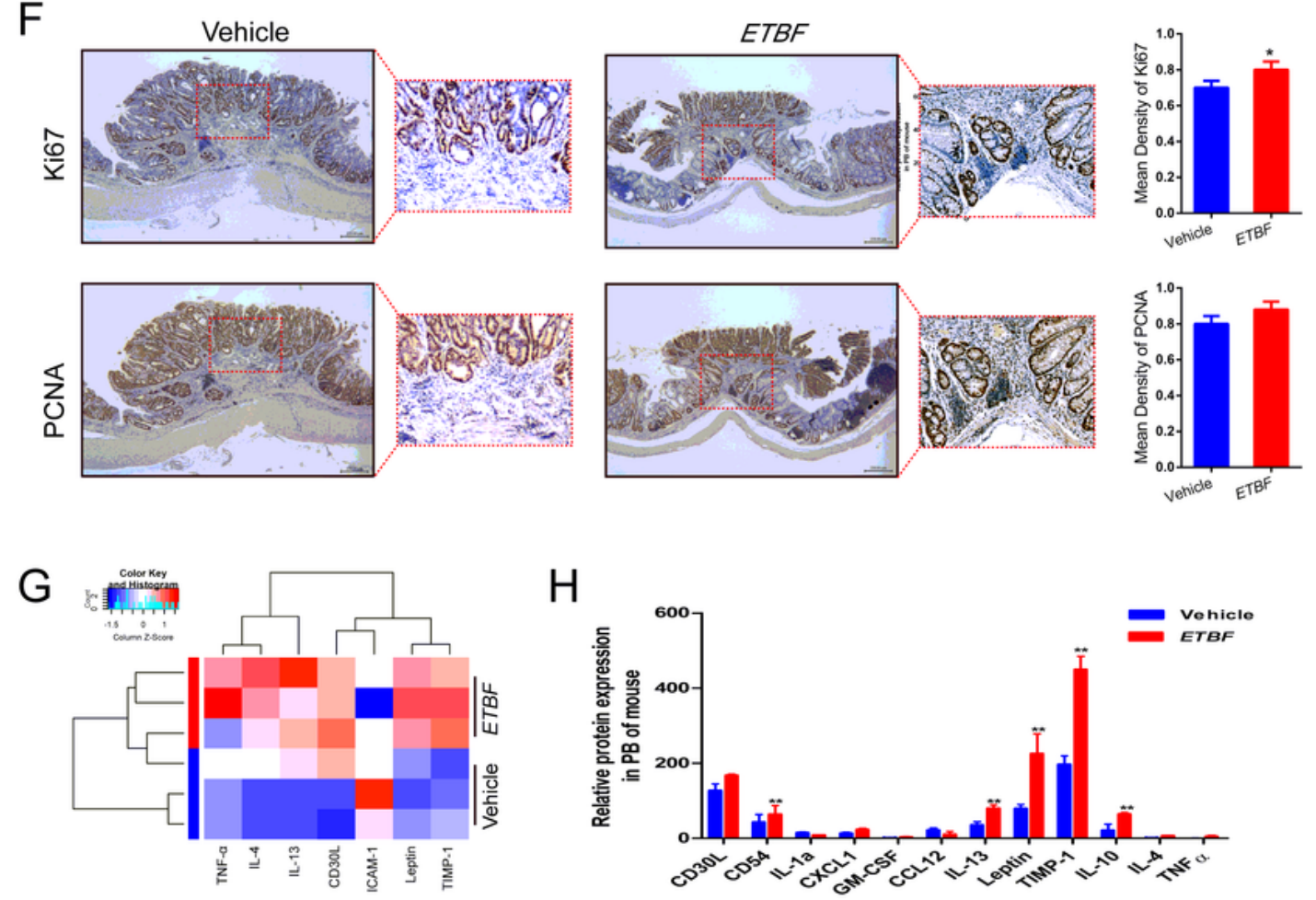

\section{Figure 3}

ETBF infection enhances colonic tumorigenesis and reduce inflammatory responses in AOM/DSS model. (A) Experimental design indicating the timing of the intragastric administration and organization of groups. Mice were treatmented with Abx from W1 (age at week 4) to W4, then injected with AOM (12.5 $\mathrm{mg} / \mathrm{kg}$, i.p.) and provided drinking water for 1 week, and three cycles with DSS and drinking water for 3 weeks as described in the Methods section. During the treatment, ETBF and Vehicle were oral 
administration with $108 \mathrm{CFU} /$ mice. (B, C) Effects of ETBF on colon length (B) and number of tumors (C) in GF/AOM/DSS mice. (D)Typical adenomatous intestinal polyp with the early invasion of neoplastic glands into the muscular layers often observed in ETBF infected GF/AOM/DSS mice. This typical regressive intestinal cancer morphology is observed throughout the intestine in the mice. Blue arrows indicated adenocarcinoma cell. Magnification bars, $100 \mu \mathrm{M}$. (E) Histology evaluation of colon tumors: quantitatively represented as polyp, adenoma, and adenocarcinoma. $(F)$ Immunohistochemical staining using an antibody against Ki67 and PCNA in the vehicle group and ETBF infection group. Magnification bars, $100 \mu \mathrm{M}$. Data are presented as the means \pm SD of 5 regions per slice with Welch's correction, onetailed t-test. ${ }^{\star \star} \mathrm{P}<0.01$, vs. Vehicle. $(\mathrm{G})$ The difference between the two groups in inflammatory cytokines as assessed by cytokine antibody array. $(\mathrm{H})$ The mRNA expression levels of inflammatory factors, such as IL-12, TNF-a, and IL-10 in the PB of mice in different groups. The data are presented as the mean \pm SD from at least three experiments. ${ }^{*} P<0.05,{ }^{*} \mathrm{P}<0.01 \mathrm{vs}$. Vehicle. 


\section{Figure 4}

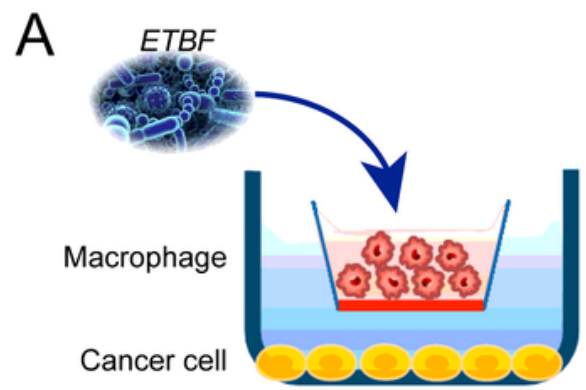

B

응

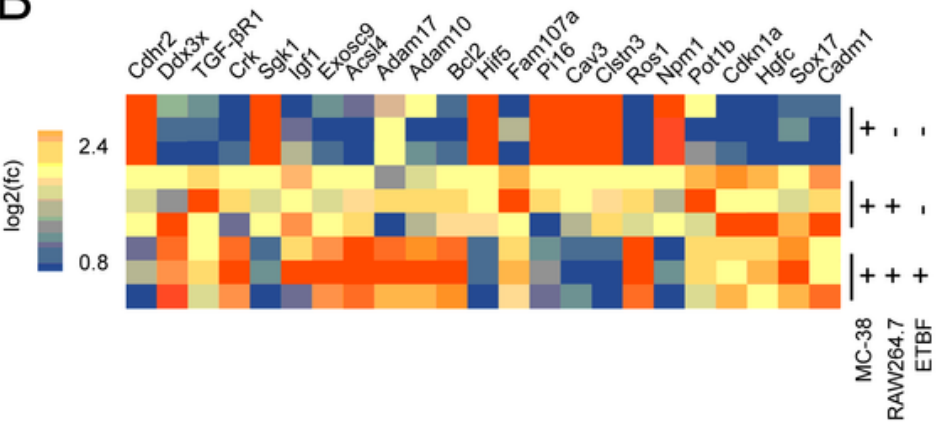

C

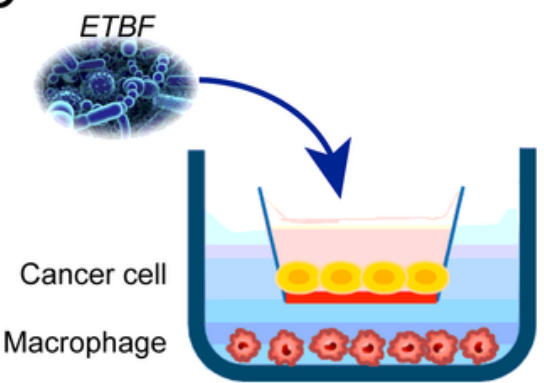

D

E
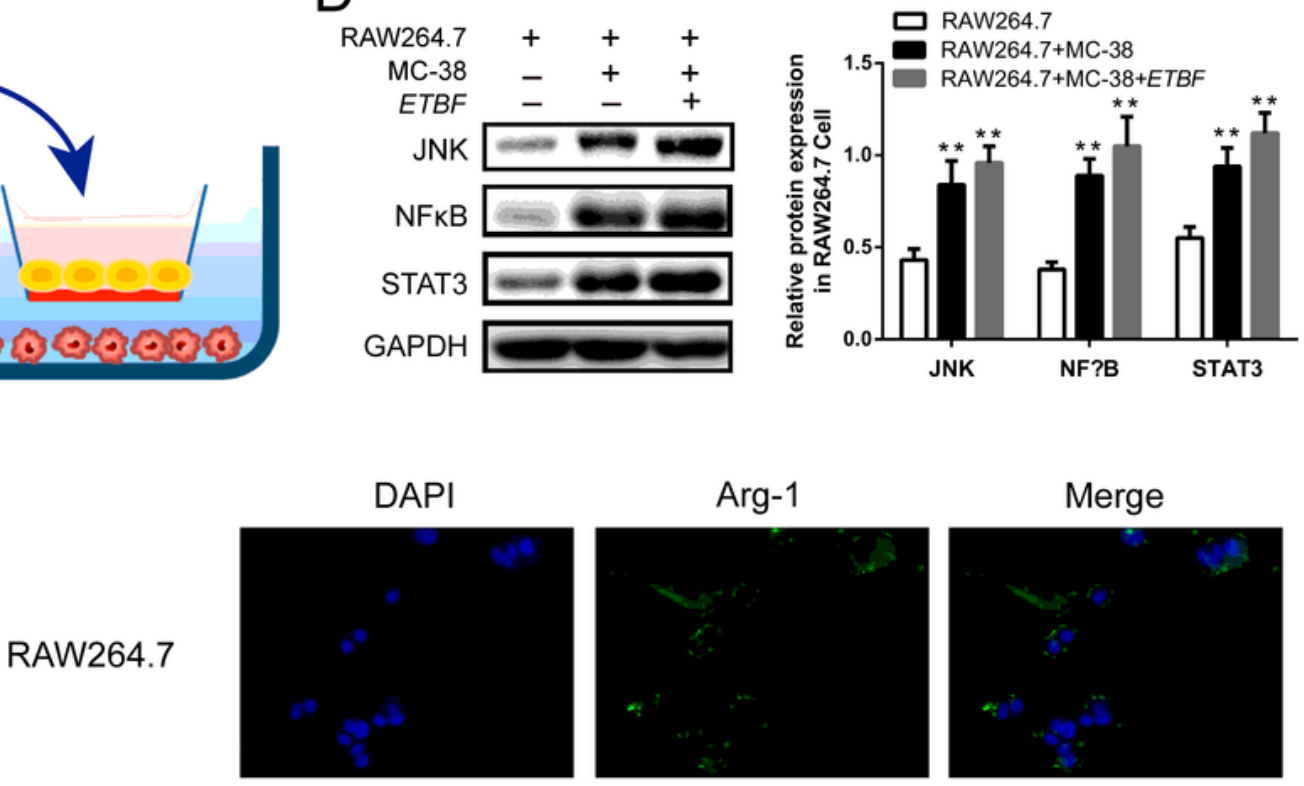

GAPDH
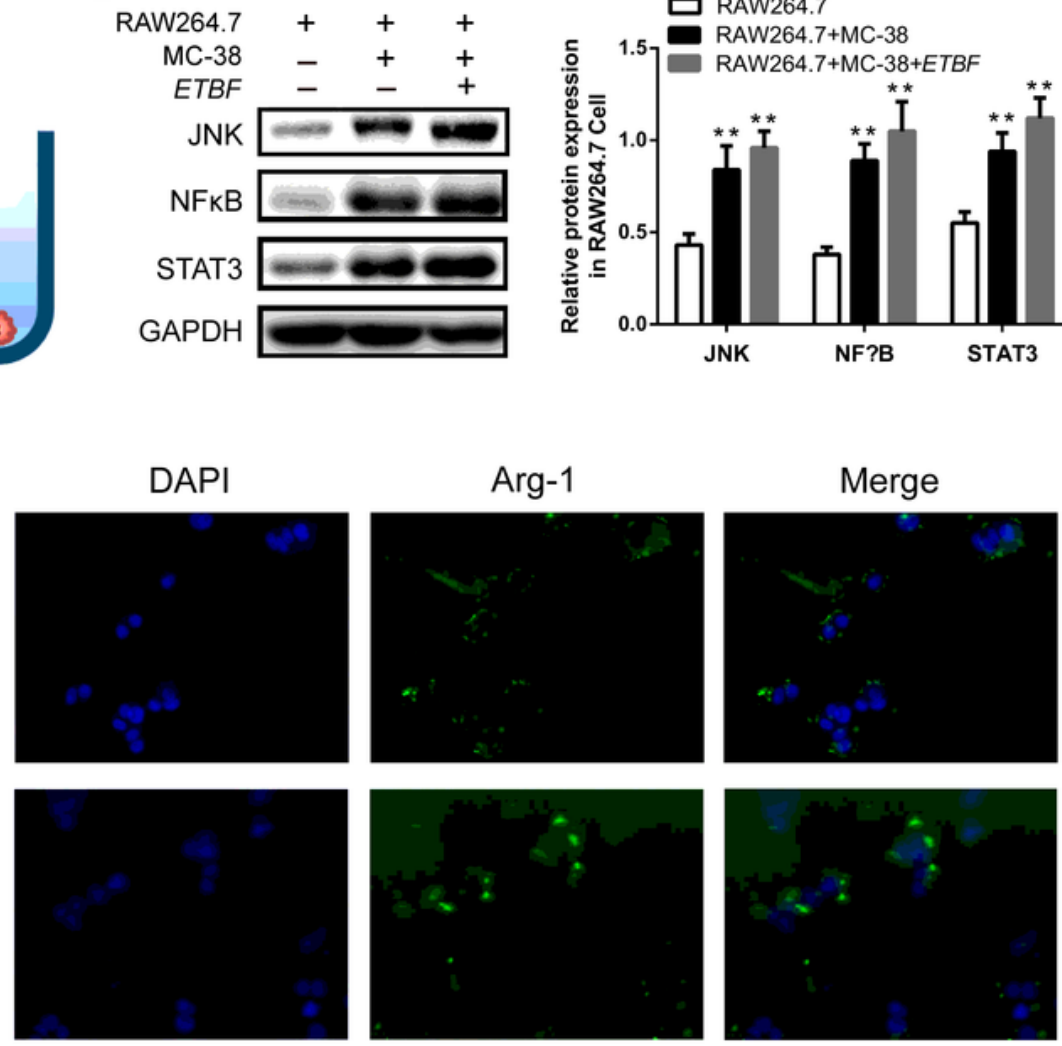

RAW264.7+MC-38

RAW264.7+MC-38+ETBF
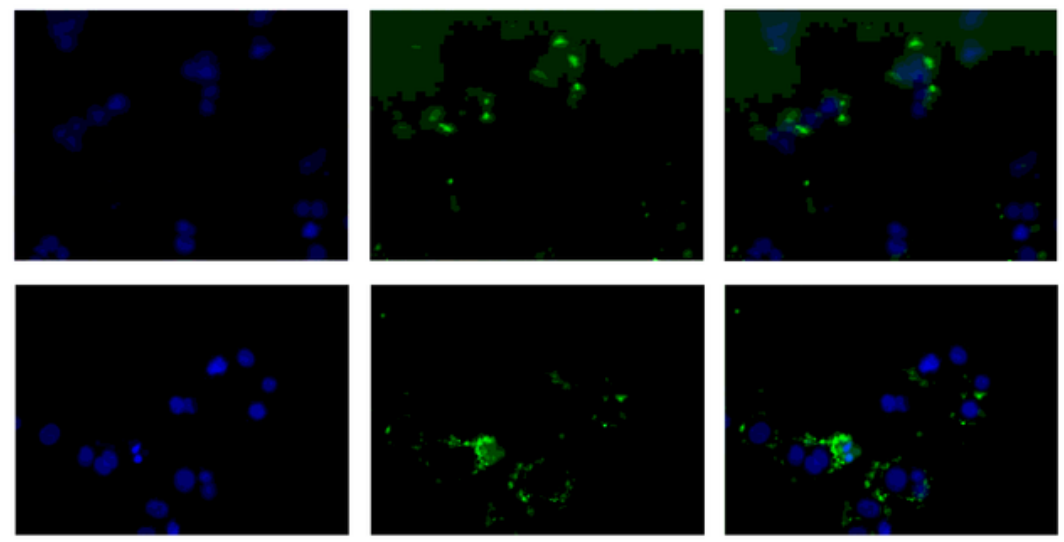

\section{Figure 4}

Effect of ETBF on CRC cells through TAMs in vitro. (A) After TAMs were co-cultured with MC-38 cells, ETBF were added into the mixture for $6 \mathrm{~h}$. The ratio of cell to bacterial was 1:10. (B) The mRNA of MC-38 cells which were at the bottom of the cell plate was analyzed by RNA-seq as described in the Methods section. (C) Experimental flow chart as Fig4A. (D) Western blot analysis of JNK, NFK-B and STAT3 expression in RAW 264.7 cells after infection with ETBF at co-cultured with MC-38 cells. Quantifications 
of migrated cells from three independent experiments are shown as the mean \pm S.D. ${ }^{*} P<0.05, * \star P<0.01$, vs. RAW 264.7 cells. (E) Immunohistochemical staining with an antibody against Arg-1 in the different group. Magnification bars, $100 \mu \mathrm{M}$.

\section{Figure 5}

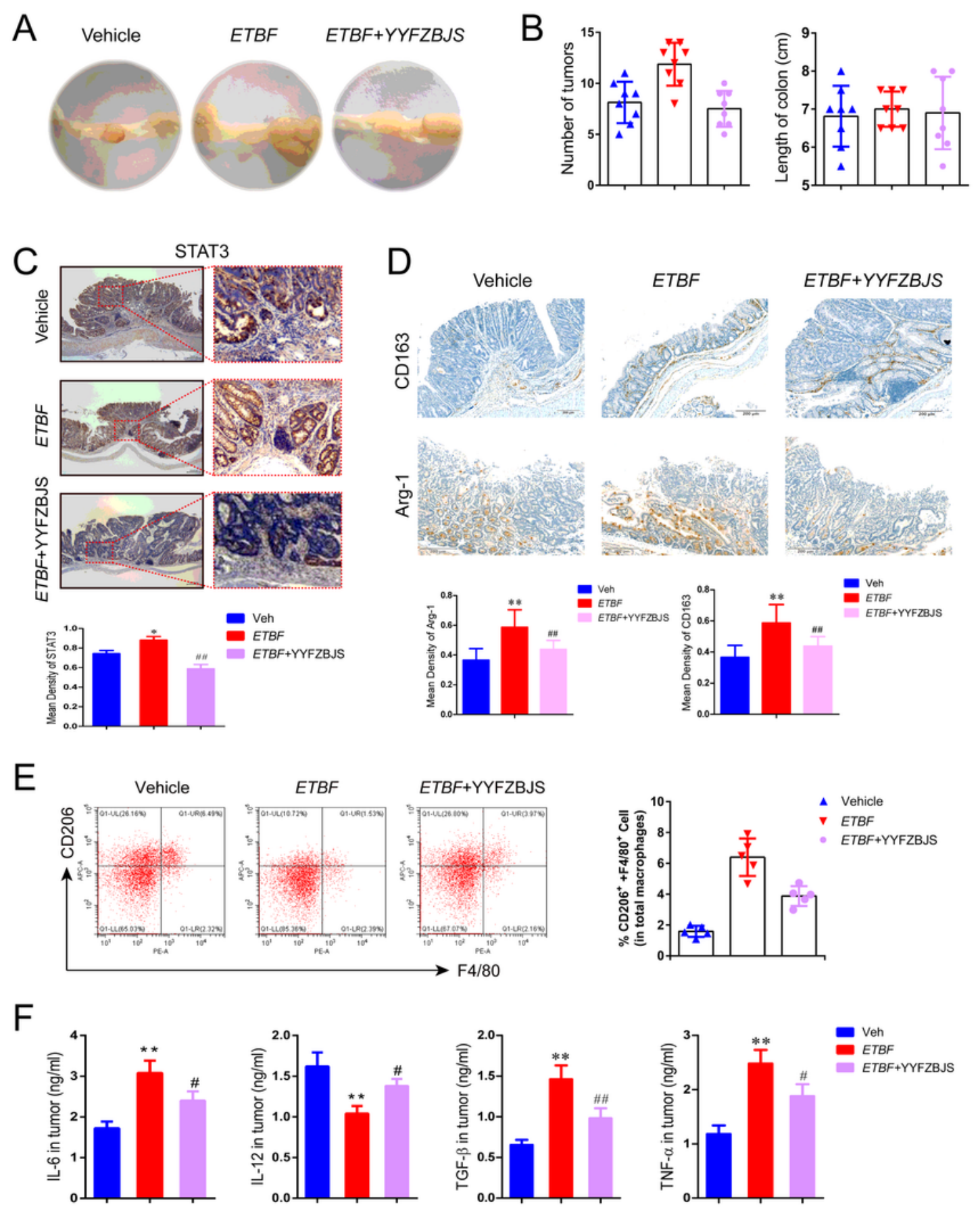

\section{Figure 5}

YYFZBJS inhibited ETBF-induced colorectal tumor development in AOM/DSS model. (A) Macroscopic view of the representative mouse large intestinal shows several polypoid and discoid colonic tumors from 
different groups of ETBF-infected mice administered with or without YYFZBJS as shown in Supplementary Fig. S1. GF/AOM/DSS mice were infected with ETBF. During ETBF infection, the YYFZBJS -treated group was orally gavaged with YYFZBJS (15.3 g/kg, p.o., once a day). (B) Tumors number and colon length distribution. (C\&D) Immunohistochemical staining with an antibody against STAT3, CD163, and Arg-1 among Vehicle group, ETBF group and ETBF+YYFZBJS group. Magnification bars, $100 \mu \mathrm{M}$. Data are presented as the means \pm SD of 8 animals per experimental group with Welch's correction, onetailed t-test. ${ }^{*} P<0.05,{ }^{\star *} P<0.01$, vs. Vehicle. (E) Representative gating strategy used to identify pulmonary macrophage subsets from intestinal tumor tissue in GF/AOM/DSS mice is shown. Macrophages were analyzed by using a flow cytometry analysis of the M2 macrophage marker CD206 in the F4/80 + cell fraction. The data are presented as the mean \pm SD of at least three experiments. (F) IL-6, IL-12, TGF- $\beta$, and TNF-a levels in Tumor were evaluated using ELISA. The data are presented as the mean \pm SD of at least three experiments. ${ }^{*} P<<0.01$, Vehicle vs. ETBF; $\# \# P<0.01$, Vehicle vs. ETBF + YYFZBJS. 


\section{Figure 6}

A

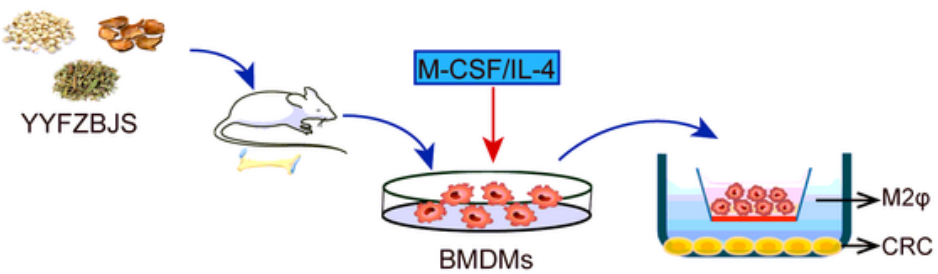

B

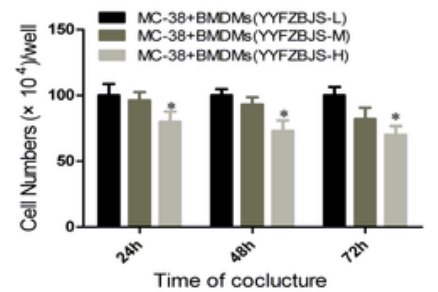

Time of coclucture

C

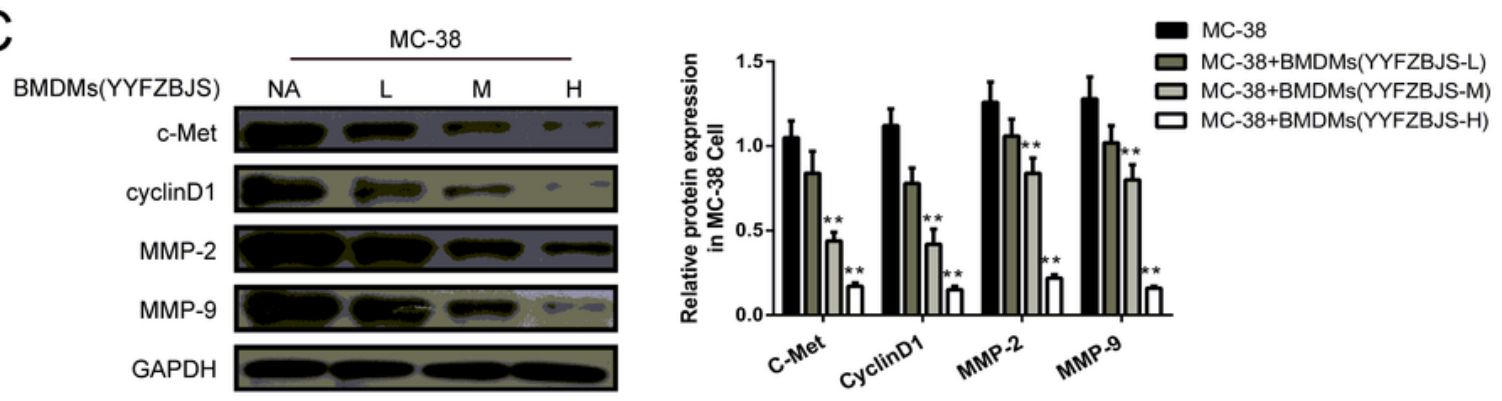

$\mathrm{D}$
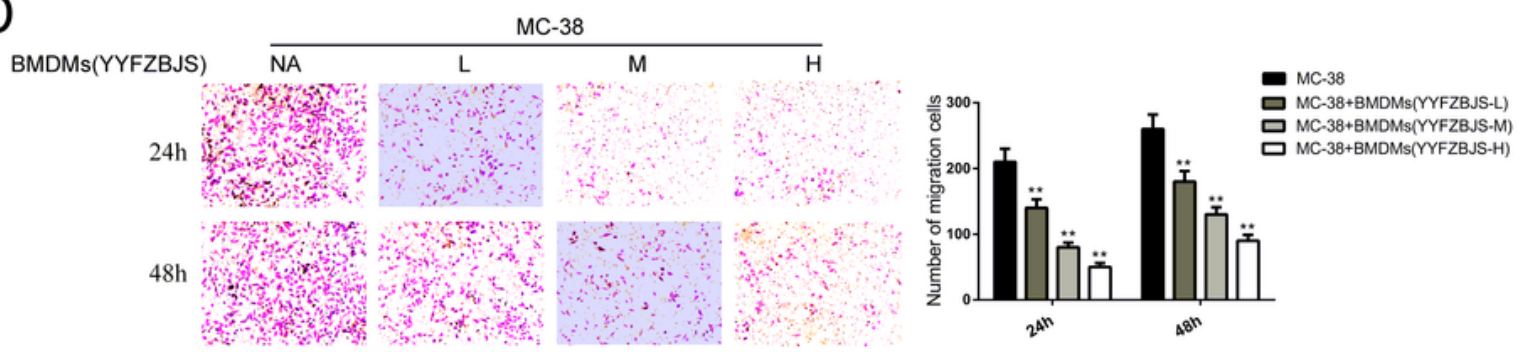

$E$

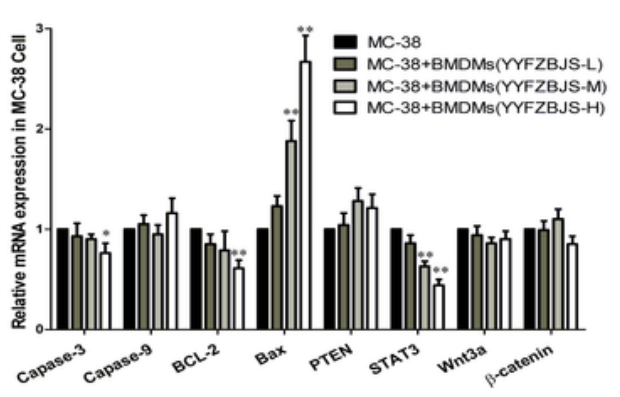

$\mathrm{F}$

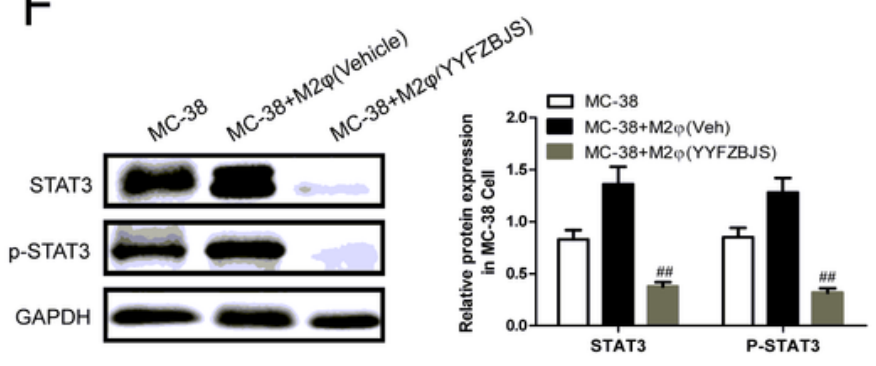

Figure 6

YYFZBJS inhibited tumor cell proliferation through regulating ETBF primed BMDMs in vitro. (A) Experimental design indicating bone marrow-derived macrophages (BMDMs) were isolated from bone marrow of C57BL/6J mice treated with or without YYFSBJS at the doses of $3.825 \mathrm{~g} / \mathrm{kg}, 7.65 \mathrm{~g} / \mathrm{kg}$ and $15.3 \mathrm{~g} / \mathrm{kg}$ for 2 weeks. The BMDMs were from femurs and tibias of mice and cultured in special medium (DMEM containing 10\% FBS supplemented with $50 \mu \mathrm{g} / \mathrm{ml}$ penicillin/ streptomycin and $10 \mathrm{ng} / \mathrm{ml}$ 
recombinant macrophage colony-stimulating factor [M-CSF; Thermo Fisher Scientific]). Then the primed BMDMs (M2 $\varphi$ ) were collected and were assigned to MC-38 cells as 10:1 ratio. (B) MC-38 cells proliferation was assayed at 24,48 , and $72 \mathrm{~h}$ after co-culture with the $\mathrm{M} 2 \varphi$. The data are presented as the mean $\pm S D$ from at least three experiments. ${ }^{*} P<0.05,{ }^{*} \mathrm{P}<0.01$ vs. MC-38+BMDMs (YYFZBJS-L). (C\&F) Western blot and quantitative assay of c-Met, cyclinD1, MMP-2, MMP-9, STAT3 and phosphorylation of STAT3 in MC-38 cells. GAPDH as loading control. The data are presented as the mean \pm SD from at least three experiments. ${ }^{*} \mathrm{P}<0.01$ vs. MC-38; \#P<0.05, \#\#P<0.01 vs. MC-38+TAM (Veh). (E) mRNA expression of genes associated with cell proliferation in MC-38 cells were evaluated using quantitative RT-PCR. The data are presented as the mean \pm SD from at least three experiments. ${ }^{*} P<0.05,{ }^{\star \star} P<0.01$ vs. MC-38.

\section{Supplementary Files}

This is a list of supplementary files associated with this preprint. Click to download.

- Supplementarydata.doc 